\title{
ENFERMEDAD INFLAMATORIA INTESTINAL EN LA DIGESTIVE DISEASE WEEK 2016: NOVEDADES EN EPIDEMIOLOGIA, SEGUIMIENTO, MONITORIZACION Y CONTROL TERAPEUTICO, Y PREVENCIÓN DEL CÁNCER DE COLON
}

\section{Autor:}

Santiago García-López, M.D., Ph. D. Servicio de Aparato Digestivo. Hospital Universitario Miguel Servet, Avda. Isabel La Católica, Zaragoza.

\section{Correspondencia:}

Santiago García López

Hospital Universitario Miguel Servet, planta 8, Digestivo.

C.P. 50009, Zaragoza, España

Teléfono: 0034-976765500 extensión 1833

E-mail: sgarcia.lopez@gmail.com

Conflictos de interés: El autor ha participado como ponente y recibido ayudas de investigación y para asistencia a congresos de Abbvie, Dr. Falk Pharma, FAES, Falk, Ferring, MSD y Shire. 
ABREVIATURAS UTILIZADAS EN EL TEXTO (orden de aparición)

\begin{tabular}{|l|l|}
\hline Digestive Disease Week & DDW \\
\hline Enfermedad inflamatoria & EII \\
\hline Cáncer de colon & CCR \\
\hline Colitis ulcerosa & CU \\
\hline Enfermedad de Crohn & EC \\
\hline Single Nucleotide Polymorphism & SNPs \\
\hline $\begin{array}{l}\text { Fermentable Oligosaccharides, } \\
\text { Pisaccharides, Monosaccharides And } \\
\text { disacáridos, monosacáridos y polioles } \\
\text { fermentables) }\end{array}$ & FODMAPs \\
\hline Infliximab & IFX \\
\hline Factor de necrosis tumoral & TNF \\
\hline Vedolizumab & VDZ \\
\hline Ustekinumab & UTK \\
\hline Crohn's disease & CDAI \\
\hline Adalimumab & ADA \\
\hline Proteína C reactiva & PCR \\
\hline Acido desoxirribonucleico & ADN \\
\hline
\end{tabular}




\section{RESUMEN}

En la Digestive Disease Week 2016 se han comunicado algunos datos interesantes, aunque de valor muy relativo, sobre un eventual papel de algunos alimentos en la enfermedad inflamatoria intestinal. También se presentaron otros datos epidemiológicos de interés, algunos sobre su historia natural. Algunos trabajos ha abordado un aspecto muy necesario para el clínico práctico, como es encontrar factores predictivos individuales. Los avances presentados son relativos, a descartar quizá un trabajo que sugiere que un parámetro tan sencillo como es la monocitosis podría ser un claro factor predictor de mala evolución (resultados muy llamativos pero con muchas limitaciones) y otro que implica a la composición corporal en la predicción de respuesta a los anti TNF. Sí hay datos más relevantes sobre la monitorización y optimización de los tratamientos biológicos, buscando su individualización. Disponemos de nuevos estudios sobre niveles de diferentes anti TNF, vedolizumab e incluso ustekinumab. También se comunicó un trabajo que pretendía estimar la seguridad de los tratamientos anti TNF según aspectos genéticos (y clínicos) del paciente. Sobre la monitorización de la enfermedad en si misma, los resultados presentados creo que no modifican nuestra práctica clínica. En la prevención del cáncer de colon los datos presentados tampoco modificarán nuestra actuación práctica clínica, pero se comunicó un estudio con una novedosa y prometedora estrategia. Se trata del uso de un nuevo panel de DNA en heces, con excelentes resultados en la detección de displasia de alto grado o cáncer colorrectal en estos pacientes.

Palabras clave: Enfermedad inflamatoria intestinal, enfermedad de Crohn, colitis ulcerosa, seguimiento, monitorización, niveles de agentes biológicos, epidemiología, prevención del cáncer de colon. 


\section{INTRODUCCIÓN}

Un año más, la Digestive Disease Week (DDW) ofrece muchísima información sobre la enfermedad inflamatoria intestinal (EII). Además de la relativa a "nuevos tratamientos", la más "vistosa", son muchos los datos presentados sobre "otros aspectos", tales como su epidemiología, optimización de terapias ya empleadas, monitorización del paciente o la prevención del cáncer de colon (CCR). Este año, en mi opinión los avances con traducción práctica no son tantos, pero intentaré recalcarlos a continuación, sobre todo aquellos con traducción práctica y que resumo en la tabla 1.

\section{¿Existe algún aVANCE RELEVANTE EN LA Etiología 0 EPIDEMIOLOGÍA DE LA ENFERMEDAD INFLAMATORIA INTESTINAL?}

Los estudios epidemiológicos en busca del descubrimiento de los factores etiológicos de la Ell se centran, un año más, en la dieta, la microbiota, la genética y su interrelación. Los trabajos relacionados con la dieta adolecen de limitaciones muy importantes, innatas a la forma de extraer los datos (encuestas de valor muy limitado), a potenciales factores de confusión (otros nutrientes como la fibra, etc.) o a su relación con la microbiota, por ejemplo, como se puso de manifiesto en todas las discusiones posteriores a las comunicaciones. Uno de ellos (1) intentó encontrar alimentos que pudieran relacionarse con exacerbaciones de la enfermedad, en concreto en la colitis ulcerosa (CU), como nos sugieren a veces los pacientes y algunas publicaciones previas $(2,3)$. Barnes y colaboradores evalúan la influencia de la dieta occidental, con exceso de carnes, proteínas y grasas saturadas, en relación al consumo de frutas y verduras, en un estudio prospectivo, observacional y multicéntrico en 412 pacientes con CU estable bajo tratamiento con mesalazina. Se identificaron muchos nutrientes asociados al desarrollo de brotes de la CU (como el aceite de coco, la lactosa a altas dosis o el ácido linolénico), aunque ningún patrón de consumo específico, como por ejemplo de carne, proteínas o alcohol. Otro estudio analizó el potencial papel del sodio y el potasio en la dieta (4), así como el papel de algunos genes. Se llevó a cabo sobre los estudios de salud de enfermería americanos, conocidos trabajos amplios en 
curso, en los casos incidentes mediante un análisis caso-control. El consumo de potasio se asoció, de manera inversa, con el desarrollo de enfermedad de Crohn (EC), asociado también a algunos SNPs concretos, relacionados con la vía de la IL-23. Otro estudio se centró en el eventual papel del hierro en la dieta (5). Se llevó a cabo también sobre los estudios de salud de enfermería, analizando el riesgo con el hierro, las carnes rojas y su relación con los genes implicados en la vía de la autofagia. No se encontró relación estadísticamente significativa entre el consumo de hierro ni carne en la Ell, aunque si alguna tendencia. Es fácil intuir que estas conclusiones son muy débiles, por las tremendas limitaciones del trabajo y que no permiten establecer ningún tipo de recomendación. Otro estudio analizó el potencial papel de la fruta (6), en este caso en el escenario concreto de la reservoritis. Sus resultados sugieren que el consumo de fruta protegió del desarrollo de reservoritis. Finalmente, otro trabajo evaluó el potencial papel del consumo de FODMAPs (7). Nuevamente las conclusiones son muy débiles, pero mostró, tras analizar 365 pacientes (201 con enfermedad de Crohn (EC) y 164 con $\mathrm{CU})$, un potencial efecto negativo de uno sólo de los FODMAPs, la lactosa, que incrementó los síntomas y la inflamación en la CU y la inflamación en la EC. Insistimos en las tremendas limitaciones de todos estos estudios.

Otros estudios epidemiológicos interesantes comunicados exploraron la historia natural de la Ell, de los que comentaré dos. El primero de ellos analizó con que frecuencia se obtiene la curación mucosa en la CU tras el año de su diagnóstico (8). En una cohorte poblacional de 6 países Asiáticos, se investigó que proporción de casos de CU incidentes (nuevos diagnósticos desde 2011 a 2014) alcanzaron la cicatrización mucosa al año de tratamiento, y que factores lo predijeron. Entre estos 154 casos nuevos de CU, al diagnóstico la actividad endoscópica fue grave (Mayo 3) en el 32\%, moderada (Mayo 2) en el $40 \%$ y leve (Mayo 1) en el $28 \%$. Al año de evolución, la cicatrización mucosa (entendida como Mayo 0 ó 1, fue del $47 \%$, encontrando una mejoría en el 44\% (Mayo 0 el 18\%)). Predijeron la cicatrización mucosa al año el sexo femenino y la menor gravedad endoscópica al diagnóstico. El segundo estudio que mencionaré, aunque no es de historia natural como tal, es también muy interesante y se describe en detalle en otro capítulo. 
Fiorino y colaboradores analizaron que sucede tras la suspensión del tratamiento anti TNF en la CU (9). Incluyeron pacientes con CU estable al menos 12 meses y sin necesidad previa de optimización de IFX y analizaron retrospectivamente la evolución posterior de dos cohortes: aquellos que discontinúan IFX frente a los que lo mantienen. La tasa de recaídas, en un seguimiento amplio, fue muy superior en los pacientes que discontinuaron el anti TNF. De las estrategias seguidas tras la discontinuación, la mejor fue el tratamiento con tiopurinas, con o sin mesalazina asociada frente a ésta sóla $(p=0,032)$. Ante una recaída, el retratamiento con IFX obtuvo la respuesta en el $77,1 \%$, aunque la remisión la alcanzó el 50\%. La tasa de reacciones infusionales fue del 17\% y una cifras muy similar de efectos adversos de otro tipo.

Conclusión práctica: No hemos avanzado apenas en encontrar la etiología de la Ell. Dicho esto, la epidemiología es una herramienta muy útil en este sentido y pone de manifiesto que los aspectos ambientales son importantes en el desarrollo de la Ell, aunque no bien conocidos. Entre ellos, la dieta y la microbiota, además de otros como los fármacos o el desarrollo en ambiente rural o urbano, podrían influir. Su interacción con la genética está también por descubrir. En los pacientes con CU estables tras 1 año con tratamiento anti TNF, retirarlo implica una elevada tasa de reactivaciones. Si se retira en algún caso, se recomienda continuar con tiopurinas.

\section{¿DISPONEMOS DE MEJORES FACTORES PREDICTORES DE LA EVOLUCIÓN INDIVIDUAL DE NUESTROS PACIENTES CON ENFERMEDAD INFLAMATORIA INTESTINAL?}

Constituye sin duda una necesidad clínica que nos permitiría ajustar mucho más individualmente el riesgo-beneficio del manejo de nuestros pacientes con Ell, pero está DDW 2016 no ha solucionado este problema.

Un estudio sugiere que un parámetro tan sencillo como es la monocitosis sería un firme factor predictor de mala evolución (10). Merece la pena destacarlo y mencionarlo porque sus resultados son muy llamativos y proviene de un grupo, como es el de Pittsburgh, que históricamente ha realizando estudios muy 
relevantes en este sentido. Este año presentan sus datos con este sencillo marcador, como es la monocitosis, células de conocido papel en la respuesta innata inadecuada a la flora intestinal. Llevan a cabo un análisis de 6 años de revisión sobre su base de datos prospectiva y analizan la evolución de los pacientes con o sin monocitosis. Los resultados son muy llamativos: aquellos con monocitosis tuvieron una clara peor evolución, con una enfermedad más grave, mayor consumo de fármacos, mayor asistencia a urgencias, mayor número de ingresos y cirugías, de forma independiente. Indudablemente hay tremendas limitaciones del estudio, como quedó patente en la discusión (por ejemplo decir que se consideró como monocitosis simplemente una cifra aislada elevada durante todo el seguimiento, o mencionar que no se analizaron sus causas en ningún momento, incluyendo el papel de algunos fármacos), pero los datos son cuando menos muy llamativos y la determinación de los monocitos es sencilla y rutinaria.

Otro trabajo que creo que es importante, por los escasos datos disponibles en el tema que aborda, analiza el uso de los servicios de urgencia por los pacientes con EII (11). Este estudio analizó con qué frecuencia acuden a urgencias los pacientes con Ell, cuales lo hacen más, cuando duran sus visitas y cuanto ingresan, además de tratar de identificar si existen factores que predicen estos aspectos. Es un estudio retrospectivo caso-control a partir de la base de datos poblacional de Manitoba, que incluyó a casi 3700 pacientes con Ell (casi 3400 casos prevalentes y 300 incidentes) y más de 21500 controles pareados por edad y sexo. Los pacientes con Ell acudieron a urgencias más que los controles, su evaluación en urgencias fue más prolongada y con una probabilidad de ingreso superior. Dentro de los sujetos con Ell, los casos incidentes lo hicieron más que los casos prevalentes. Como factores predictores de la asistencia a urgencia se encontraron la duración de la enfermedad y el uso de esteroides y narcóticos. La duración de la visita en urgencias fue superior a los controles, y mayor cuando el motivo era sintomatología "intestinal", y la frecuencia con la que fueron ingresados fue superior. Todos estos aspectos fueron más acusados en los casos "incidentes" (en proceso diagnóstico de la enfermedad o en su periodo inicial de tratamiento). Los 
pacientes tratados con anti TNF acudían a urgencias tanto como el resto, pero ingresaban menos. Es un estudio con múltiples limitaciones, entre ellas que no sabemos si estas visitas a urgencias fueron razonables o no, así como los ingresos correctamente indicados o no.

Conclusión práctica: No disponemos de nuevos factores aplicables a la clínica en este momento que permitan predecir la evolución de un paciente concreto. La monocitosis o la composición corporal podrían ser factores implicados. Los casos de Ell recién diagnosticados probablemente hacen un mayor uso de los servicios de urgencias que quizá podríamos optimizar.

\section{¿QUE HAY DE NUEVO EN EL SEGUIMIENTO I MONITORIZACION DE LA ENFERMEDAD INFLAMATORIA Y DE SUS TRATAMIENTOS?}

Sobre la monitorización de la enfermedad en si misma, en mi opinión no hay resultados que modifiquen nuestra práctica clínica. Mencionaremos un solo estudio sobre ecografía, metodológicamente bien realizado. Sí hay datos más interesantes sobre la monitorización, optimización e individualización de los tratamientos biológicos, sobre los que se han comunicado nuevos estudios con datos especialmente relevantes sobre niveles de los diferentes anti TNF, además de sobre vedolizumab (VDZ) e incluso sobre ustekinumab (UTK). También se comunicó un trabajo que pretende estratificar la seguridad de los tratamientos según determinantes genéticos del paciente.

\section{Seguimiento de la Ell}

La importancia potencial de la ecografía es evidente, pero no disponemos de índices validados y sencillos. Además, los que hay suelen cuantificar el daño intestinal mas que la actividad (12). Un trabajo de la DDW 2016 desarrolla un nuevo índice de ecografía, sencillo y con validación posterior (13). En una cohorte de 161 pacientes desarrolla el índice, mediante una comparación por segmentos frente a la endoscopia. Posteriormente valida externamente el índice en cohorte prospectiva de 63 enfermos. Incluye como parámetros ecográficos el engrosamiento de la pared, ecogenicidad de la grasa mesentérica, ganglios 
mesentéricos, hiperemia, además de las complicaciones. El parámetro ecográfico más importante como predictor de la actividad fue el engrosamiento de la pared. La importancia del control de la nutrición quedó patente en otro estudio multicéntrico español (14), que investiga no sólo aspectos muy detallados del estado nutricional en la Ell, sino también que razones y causas subyacen. El estudio fue prospectivo e incluyó a más de 1200 pacientes con EC y CU atendidos consecutivamente en las unidades participantes. La prevalencia de malnutrición fue del 28\%. Una fuerza muscular por debajo del percentil 10 se encontró en el $10 \%$ de los pacientes. Los factores asociados al riesgo de malnutrición fueron una historia de cirugía abdominal previa, la Ell activa o evitar alimentos durante un brote de la actividad. La mitad de los pacientes creen que algunos alimentos les pueden desencadenar un brote, casi el $70 \%$ modifican sus hábitos alimenticios tras el diagnostico e incluso casi el $90 \%$ evitan algunos alimentos para prevenir los brotes.

\section{Monitorización, optimización e individualización del tratamiento de la Ell}

Me centraré esencialmente en las terapias biológicas. Revisaré a continuación algunos estudios comunicados cuyo objetivo es individualizar el tratamiento mediante: identificación de los mejores candidatos a recibir estas terapias (mayor probabilidad de respuesta y menor probabilidad de efectos secundarios) y maximizar su eficacia. La Dra. Chaparro aborda en su capítulo algunos trabajos también interesantes en este mismo sentido, cuya lectura recomiendo.

a) Identificación de los mejores candidatos a terapias biológicas:

Constituiría indudablemente una excelente forma de optimizar su uso, no conseguido hasta la fecha, ni solucionado por esta $D D W$, aunque si se comunicaron algunos datos en ese sentido. Así, en la búsqueda de factores predictivos de respuesta, una comunicación oral sugería que la composición corporal podría ser uno de ellos (15). Ding y colaboradores evalúan la evolución de los pacientes y su respuesta a los anti TNF de acuerdo a la composición corporal (no el índice de masa corporal, sino la composición en músculo y grasa). 
Sorprendía que dicha medición se realizó mediante tomografías computarizadas (TCs) periódicas. En cualquier caso, la miopenia se relacionó con una peor respuesta primaria a anti TNF. Sugieren que se podría utilizar para una mejor dosificación del anti TNF, la masa libre de grasa. Otro factor, menos discriminativo, podría ser el sexo, de forma que un póster sobre una cohorte en Holanda sugiere que las mujeres podrían tener una peor respuesta a anti TNF, al menos a largo plazo (16). En cualquier de los casos, parece que el fallo primario a los anti TNF es un factor de mal pronóstico futuro en la Ell (17). La otra forma de optimizar los tratamientos sería conocer sus riesgos antes de iniciarlos. Así, una comunicación oral evaluó la seguridad de diversos tratamientos, buscando determinantes clínicos o genéticos predictores individuales de efectos secundarios (18). En la cohorte de pacientes con Ell de su centro hospitalario (Michigan; cohorte PRISM), realizaron estudio genético de determinados SNPs potencialmente relacionados, y análisis de factores clínicos. De los 1333 pacientes con Ell, 133 habían presentado ingresos relacionados con infecciones, aunque ninguna fatal. Fueron más frecuentes en la EC, sobre todo de patrón penetrante. Los esteroides, inmunomoduladores y biológicos no se asociaron con mayor riesgo de infecciones. Nueve SNPs si aumentaron el riesgo, 2 de ellos de forma evidente, siendo posible elaborar un índice de riesgo genético predictivo de infecciones. Analizaron los factores clínicos por separado y en combinación al índice genético, comprobando que éstos mejoraron la estimación basal del riesgo individual de infecciones ante los tratamientos. Entre las limitaciones del estudio destacar el tamaño muestral, que no permitió revisar el efecto de la combinación de fármacos o no haber analizado específicamente la gravedad de la infección, ni los "tiempos" en que se produjeron.

b): maximizar su eficacia: niveles y su optimización en diferentes escenarios: Los datos más relevantes son con anti TNF y dentro de ellos, el estudio quizá más importante es el llamado TAILORIX (19). Se trata de un ensayo randomizado y controlado en la EC luminal activa, cuyo objetivo es investigar si el tratamiento dirigido ("tailor") por niveles, con inflximab ("ix") (IFX) en combinación con 
inmunosupresores, es así más eficaz, llevado a cabo en varios centros en Holanda, Bélgica y Francia. Todos los pacientes presentan una EC activa (CDAI > 220), con datos objetivos de inflamación (PCR y/o calprotectina elevadas) y úlceras visibles en la endoscopia, y son näive a anti TNF. Los pacientes se randomizan en 3 ramas, cada una de 40 pacientes previstos, dos de control por niveles, con diferente protocolo, y una con placebo. La variable primaria de respuesta es la remisión sin esteroides en todas las visitas entre las semanas 22 y 54 , junto con la ausencia de úlceras en semana 52-54. Se incluyeron finalmente 122 pacientes y no hubo diferencias entre los 3 grupos de tratamiento en la variable primaria, así como tampoco en otras variables secundarias, como la existencia de úlceras o la necesidad de intensificación (lógicamente ésta se produjo más frecuentemente por reactivación clínica en los pacientes tratados sin ajuste por niveles). En un interesante y metodológicamente bien realizado estudio, Tedesco y colaboradores analizaron la importancia de las dosis de azatioprina en el tratamiento combinado. En pacientes en remisión profunda, näive a inmunosupresores e IFX previamente, la reducción de azatioprina a la mitad no supuso, al año de seguimiento, cambios en la tasa de reactivación o un empeoramiento en los niveles de IFX (20). Por el contrario, la retirada de azatioprina si se asoció a una disminución de los niveles de IFX, aunque no a una mayor tasa de reactivaciones. Otros trabajos, aunque no de un diseño metodológico tan adecuado, exploran el valor de los niveles en escenarios concretos y con resultados relevantes. Así, un se comunicó uno de los estudios más importantes en la utilidad de los niveles en la enfermedad perianal (21), situación en la que es especialmente importante optimizar la eficacia de los anti TNF. Se trata de un estudio observacional multicéntrico, basado en sus registros prospectivos, e incluyó pacientes con enfermedad perianal activa, con al menos 14 semanas de tratamiento y niveles de anti TNF en las 4 semanas "circundantes". La variable principal del estudio fue la cicatrización de las fístulas (presentes pero sin drenaje espontáneo ni a la presión) y una de las secundarias su cierre completo (desaparición de la fístula). Incluyeron 117 enfermos, 62\% en tratamiento combinado, y se comprobó una buena correlación entre los niveles y cicatrización 
de las fístulas, aunque no con su cierre completo. Los pacientes que cicatrizaron sus fístulas tuvieron niveles de IFX muy superiores a los que no (18,5 vs 6.5 microgramos $/ \mathrm{ml} ; \quad \mathrm{p}<0,0001)$. Niveles superiores a 10 fueron un factor independiente para obtener la cicatrización de las fístulas, siendo precisos niveles muy elevados en algunos casos. La conclusión de este trabajo sería que la monitorización de los niveles podría ser muy útil en la EC perianal, siendo posible quizá optimizar su eficacia. Existen limitaciones, una vez más algunas patentes en la discusión tras la comunicación, como el diseño retrospectivo y la ausencia de pruebas de imagen, o que no se analizó si habían incrementado las dosis tras conocer los niveles, y si así fue, si ello había sido eficaz o no. Otra comunicación analizó la utilidad de los niveles anti TNF en la gestación (22). Se trata de un estudio observacional (no se realizaron cambios ante los resultados), ciego, que incluyó gestantes con Ell estables en tratamiento con anti TNF, evaluando sus niveles (15 mujeres con IFX y 11 con adalimumab (ADA)). La concentración de IFX cambió durante el embarazo (no se ajustó la dosis al peso en cada momento), aumentado progresivamente, mientras que la de ADA se mantuvo estable. En cada grupo, 5 mujeres recibían inmunomoduladores, sin encontrar diferencias en las concentraciones del anti TNF. Este estudio viene a sugerir que podría ser razonable medir los niveles durante la gestación. Quizá podría ser útil medir los niveles antes de la gestación y utilizarlos como referencia durante la misma.

Con otros biológicos también se presentaron datos nuevos. Sobre VDZ se comunicó un análisis post hoc sobre el estudio GEMINI 1, evaluando si los niveles precoces de VDZ se correlacionan con su eficacia posterior (23). Los niveles en semana 6 se correlacionaron con la remisión clínica en semana 14 y parecen predictivos de la evolución en semana 52. De todos los pacientes tratados con VDZ, sólo el 3,7\% presentaron anticuerpos contra VDZ en algún momento, y un $1 \%$ en dos o más determinaciones hasta la semana 52 . No se estratificaron por asociación o no con inmunomoduladores, ni se disponía de datos sobre la albúmina. En conclusión, los niveles en semana 6, podrían identificar de forma precoz a pacientes en tratamiento con VDZ y aún sintomáticos que van a responder probablemente más adelante, evitando retirar el tratamiento. También 
podría reducir la necesidad de una dosis adicional en semana 10 (24). El tamaño de la muestra es pequeño y por lo tanto no se pueden extraer recomendaciones fuertes, aunque si sugiere que necesitamos más estudios. Sobre UTK se presentó un trabajo cuyo objetivo fue analizar la correlación entre sus niveles de UTK y la situación clínica, bioquímica y endoscópica (25). Los pacientes incluidos fueron todos refractarios a anti TNF y se analizaron 2 cohortes, una prospectiva ( $n=24$, se realizan niveles y analiza eficacia en semanas 10 y 26) y otra retrospectiva $(n=35)$, cuyos resultados se combinan cuando es conveniente estadísticamente. UTK obtuvo una eficacia importante, pero sólo quería destacar aquí los resultados sobre niveles y anticuerpos. Así, niveles elevados de UTK consiguieron mejores tasas de normalización de PCR y mayor eficacia endoscópica. El uso de inmunomoduladores no modificó, en este trabajo, los niveles de UTK. No se identificaron anticuerpos contra UTK en ningún caso. Son datos indudablemente preliminares y con limitaciones, pero interesantes y prometedores.

Conclusión práctica: los niveles de anti TNF (y sus anticuerpos) son útiles para tomar decisiones en la práctica clínica ante la pérdida de respuesta secundaria, pero aún no han probado su utilidad como monitorización proactiva o como factor pronóstico. En el caso de la enfermedad perianal podrían ser necesarios niveles más elevados. Disminuir o discontinuar la azatioprina al año de tratamiento combinado (anti TNF y azatioprina), en pacientes estables y en remisión profunda, es una alternativa a explorar. Disponemos de nuevos datos sobre niveles de otros biológicos, aunque no modifican nuestra práctica clínica actual.

\section{¿EXISTEN AVANCES PRÁCTICOS IMPORTANTES EN LA PREVENCIÓN DEL CÁNCER DE COLON EN LA ENFERMEDAD INFLAMATORIA INTESTINAL?}

En esta DDW 2016 se dedicaron varias mesas de debate y repaso a la prevención del CCR en la Ell, que han puesto de manifiesto que no es ni mucho menos un tema resuelto, incluso en las bases conceptuales que lo sustentan. Las recomendaciones actuales dejan atrás el concepto "displasia no visible", que justificaba las biopsias aleatorias, confiando la prevención a visualizar la displasia, mediante la cromoendoscopia. Sin embargo quizá la pregunta realmente clave no 
está tan clara... ¿estamos disminuyendo el CCR con esta estrategia preventiva en la EII?. La historia de la prevención en otros cánceres, como la mamografía en el cáncer de mama o el PSA en el de próstata, deberían obligarnos a ser cautos en nuestras recomendaciones. Desafortunadamente, a pesar de lo interesante de estas sesiones, en la DDW 2016 no se han comunicado demasiados datos nuevos, aunque si son algunos relevantes.

Uno de los trabajos más interesantes y novedosos en mi opinión, viene del uso de técnicas moleculares. Se trata de un estudio que tiene como objetivo evaluar la utilidad de la medición en heces de un nuevo panel de ADN metilado para detectar CCR o displasia (26). Es un trabajo multicéntrico con 3 poblaciones independientes, de metodología muy correcta, que analiza las muestras centralmente y de forma ciega, obteniendo la sensibilidad y especificidad en relación a la colonoscopia, en diferentes grupos de pacientes con Ell. El test alcanzó una excelente sensibilidad y especificidad para detectar CCR o displasia de alto grado, que no varió entre los grupos con distinto riesgo basal, con una tasa muy baja de falsos positivos. Los datos no son tan favorables en la displasia de bajo grado. En mi opinión se trata de unos excelentes resultados y de una estrategia muy prometedora para la detección de CCR o displasia de alto grado en la Ell. Otro trabajo comunicado pretendía evaluar el riesgo individual de CCR (y otros cánceres), y la implicación en su génesis no sólo de aspectos relacionados con la enfermedad, sino también de los tratamientos o pruebas diagnósticas radiológicas (27). El estudio, realizado en Asia, identificó los pacientes con diagnóstico de Ell en el registro de Hong Kong entre los años 1990 y 2014, que posteriormente presentaron un cáncer. Se analizaron los datos demográficos y relativos a la Ell, tratamiento y procedimientos radiológicos. La exposición radiológica se consideró como elevada si había superado los $50 \mathrm{mSv}$ y la exposición a los diferentes fármacos de forma dicotómica. Se incluyeron casi 2500 pacientes en el análisis definitivo, 960 con EC y 1472 con CU, ambos grupos con amplio seguimiento, con más de 22500 personas-año. Los sujetos con EC recibieron mayores dosis de radiación y existían diferencias en el tratamiento médico de EC y CU. Se encontraron cánceres de diferente tipo y localización, 
como cabía esperar en una población y seguimiento amplios. No se objetivó un incremento en el riesgo global de cáncer en la CU y si en la EC, que presentó un exceso neto de riesgo del $48 \%$. Este riesgo fue debido a un aumento en el riesgo de CCR en varones y también de cáncer de intestino delgado en jóvenes con afectación de tramos altos. Los pacientes con Ell y elevada exposición a radiación tuvieron un mayor riesgo de cáncer, tanto CCR como extra-digestivo. El uso de mesalazina se mostró como factor protector de CCR y también de cáncer extraintestinal, en CU y EC. Este trabajo tiene limitaciones evidentes por su compleja metodología, con muchos factores de confusión posibles o no analizados, como fue por ejemplo el tabaquismo, no controlado en el análisis, y muy criticado en la discusión posterior. No obstante, estos datos sugieren una vez más que el riesgo de cáncer no es el mismo entre todos los pacientes con Ell. La relevancia de estos datos se pone de manifiesto teniendo en cuenta los de otro estudio Canadiense que sugiere que la mortalidad por CCR en la Ell no ha disminuido en los últimos 25 años (28). Este estudio caso control, aunque con las limitaciones propias por ser realizado sobre registros y bases de datos, fue poblacional y quedó patente su valor para el objetivo que se planteó. Analizó el riesgo de los pacientes con Ell de desarrollar CCR y de morir por ello, en 3 periodos: 1987-1993, 1994-2001, 2002-2013. No se apreció una disminución firme del riesgo de CCR en la Ell, ni tampoco se ha reducido su mortalidad.

Conclusión práctica: Un nuevo método molecular de detección de ADN metilado en heces parece prometedor en la prevención del CCR en la Ell. No obstante, seguir las recomendaciones de las guías actuales es la mejor manera de intentar prevenir el CCR en nuestros pacientes con Ell. Considerar su riesgo, y en lo posible estratificarlo, aunque no dispongamos de factores bien definidos, es un objetivo en la práctica clínica. Finalmente, no sólo existe el CCR, de forma que debemos tener presentes otros potenciales tumores en la Ell, como el cáncer de intestino delgado. El uso adecuado de las técnicas radiológicas podría ayudar en su prevención. 


\section{BIBLIOGRAFÍA}

1. Edward L. Barnes, Molly A. Nestor, Louisa Onyewadume, Punyanganie S. De Silva, Joshua R. Korzenik. A Prospective Study: The Role of Diet in Exacerbations of Patients With Ulcerative Colitis in Remission on Monotherapy With Mesalamine.. Gastroenterology, Vol. 150, Issue 4, S5-S6.

2. Yates CM, Calder PC, Ed Rainger G. Pharmacology and therapeutics of omega3. polyunsaturated fatty acids in chronic inflammatory disease. Pharmacol Ther. 2014;141(3):272-82.

3. Barbalho SM, Goulart R de A, Quesada K, Bechara MD, de Carvalho A de CA. Inflammatory bowel disease: can omega-3 fatty acids really help? Annals of Gastroenterology: Quarterly Publication of the Hellenic Society of Gastroenterology. 2016;29(1):37-43.

4. Hamed Khalili, Sakshi Malik, Amit Awasthi, Ashwin N. Ananthakrishnan, John Garber, Leslie M. Higuchi, Amit D. Joshi, Joanna Peloquin, and others. Dietary Sodium and Potassium Intake, Immune Tolerance and Risk of Crohn's Disease and Ulcerative Colitis. Gastroenterology, Vol. 150, Issue 4, S19-S20

5. Hamed Khalili, Punyanganie S. De Silva, Ashwin N. Ananthakrishnan, Paul Lochhead, James Richter, Jenny Sauk, Andrew T. Chan. Dietary Iron and Red Meat Consumption, Autophagy, and Risk of Crohn's Disease and Ulcerative Colitis. Gastroenterology, Vol. 150, Issue 4, S20-S21

6 Lihi Godny, Nitsan Maharshak, Lior Yahav, Naomi Fliss Isakov, Uri Gophna, Hagit Tulchinsky, Iris Dotan. Fruit Consumption May Protect Against the Development of Intestinal Inflammation via Modification of Microbial Composition. Gastroenterology, Vol. 150, Issue 4, S21.

7. Kathy Vagianos, Kathryn Sexton, Matthew Bernstein, Pingzhao Hu, Kaiqiong Zhao, Charles N. Bernstein, Laura Targownik. Dietary Lactose Consumption Is 
Associated With Both Increased Symptoms and Intestinal Inflammation in IBD. Gastroenterology, Vol. 150, Issue 4, S41-S42

8. May Leung, Whitney Tang, Gani Niamul, Yu-Fang Wang, Qin Ouyang, Hon Ho $\mathrm{Yu}, \mathrm{Ka}$ Kei Ng, Jinwen Zhang, and others. Mucosal Healing in Ulcerative Colitis in the First Year of Diagnosis: Results From a Population-Based Inception Cohort Study From Six Countries in Asia. Gastroenterology, Vol. 150, Issue 4, S7-S8

9. Gionata Fiorino, Pierre Ellul, Martina Muscat, Pantelis Karatzas, Marco Silva, Armando Peixoto, Carla Felice, Fabrizio Bossa, and others. Infliximab Discontinuation Is Associated With a Higher Risk for Relapse in Patients With Ulcerative Colitis in Remission: A Multinational Collaborative Retrospective Study. Gastroenterology, Vol. 150, Issue 4, S6

10. Cynthia Cherfane, Alyce J. Anderson, Claudia Ramos Rivers, Marc Schwartz, Arthur Barrie, Jana G. Hashash, Miguel Regueiro, Michael A. Dunn, and others. Is Monocytosis a Biomarker of Severity in Inflammatory Bowel Disease? Analysis of a 6 Year, Prospective Natural History Registry. Gastroenterology, Vol. 150, Issue 4, $\mathrm{S} 42$

11. Zoann Nugent, Harminder Singh, Laura Targownik, Trevor Strome, Carolyn Snider, Charles N. Bernstein. Emergency Department Use by Persons With IBD; A Population Based Study. Gastroenterology, Vol. 150, Issue 4, S6

12. Panés J, Bouzas R, Chaparro M, García-Sánchez V, Gisbert JP, Martínez de Guereñu B, Mendoza JL, Paredes JM, Quiroga S, Ripollés T, Rimola J. Systematic review: the use of ultrasonography, computed tomography and magnetic resonance. imaging for the diagnosis, assessment of activity and abdominal complications of Crohn's disease. Aliment Pharmacol Ther. 2011 Jul;34(2):125-45.

13. Kerri L. Novak, Remo Panaccione, Subrata Ghosh, Gilaad Kaplan, David H. Bruining, Stephanie Wilson. A Simple, Validated Ultrasonographic Score for the Detection of Inflammation in Established Crohn's Disease. Gastroenterology, Vol. 150, Issue 4, S213 
14. María José Casanova, María Chaparro, Begoña Molina, Olga Merino, O K Nuevo-Siguairo, C Dueñas-Sadornil, A M García-Albert, Daniel Hervías, and others. Prevalence of Malnutrition and Nutritional Characteristics of Patients With Inflammatory Bowel Disease (IBD). Gastroenterology, Vol. 150, Issue 4, S89

15. Nik S. Ding, George Malietzis, Philip F. Lung, Wai M. Ylp, Lawrence Penez, Thenugan Ganeshanathan, Simon M. Gabe, John T. Jenkins, and others. Body Composition Profile: A Predictor of Therapeutic Outcome in Patients With Moderate to Severe Crohn's Disease. Gastroenterology, Vol. 150, Issue 4, S48S49

16. Mitchell R. Lie, Joany E. Kreijne, Christien Janneke van der Woude. Gender Differences in Adalimumab Continuation Rates, Results of a Prospective Crohn's Disease Cohort. Gastroenterology, Vol. 150, Issue 4, S809

17. Sine Buhl, Casper Steenholdt, Märta K. Borghede, Maria Rasmussen, Jorn Brynskov, Ole Thomsen, Mark A. Ainsworth. Long-Term Outcomes After Primary Infliximab Treatment Failure in Patients With Inflammatory Bowel Disease. Gastroenterology, Vol. 150, Issue 4, S809

18. Saranya Sasidharan, Vijay Yajnik, Hamed Khalili, John Garber, Comas Giallourakis, Ramnik Xavier, Ashwin N. Ananthakrishnan. Genetic Risk Factors for Serious Infections in Inflammatory Bowel Diseases. Gastroenterology, Vol. 150, Issue 4, S224-S225

19. Geert R. D'Haens, Severine Vermeire, Guy Lambrecht, Filip J. Baert, Peter Bossuyt, Maria Nachury, Anthony Buisson, Yoram Bouhnik, and others. Drug-Level Based Dosing Versus Symptom-Based Dose Adaptation in Patients With Crohn's Disease: A Prospective, Randomized Multicenter Study (TAILORIX). Gastroenterology, Vol. 150, Issue 4, S143).

20. Emilie Del Tedesco, Stephane Paul, Hubert Marotte, Camille Jarlot, Nicolas Williet, Jean Marc Phelip, Laurent Peyrin-Biroulet, Jean-Frederic Colombel, and others. Azathioprine Dose Reduction in Inflammatory Bowel Disease Patients on 
Combination Therapy: A Prospective Study. Gastroenterology, Vol. 150, Issue 4, S143-S144

21. Andres Yarur, Vikram Kanagala, Daniel Stein, Frank Czul, Dilpesh Agrawal, Yelena Zadvornova, Maria A. Quintero, Maria T. Abreu. Higher Infliximab Trough Levels Are Associated With a Higher Rate of Perianal Fistula Healing in Patients With Crohn's Disease. Gastroenterology, Vol. 150, Issue 4, S105-S106

22. Cynthia H. Seow, Yvette Leung, Elnaz Ehteshami Afshar, Divine Tanyingoh, Gurmeet K. Bindra, Michael J. Stewart, Paul L. Beck, Gilaad Kaplan, and others. Pregnancy Alters the Pharmacokinetics of Infliximab and Adalimumab in IBD Women. Gastroenterology, Vol. 150, Issue 4, S105.

23. Mark T. Osterman, Xavier Roblin, Sarah C. Glover, Udayakumar Navaneethan, Mihaela A. Popa, Tim Wyant, Alexandra James, Karen Lasch, and others.Association of Vedolizumab Drug Concentrations at or Before Week 6 With Remission at Week 14 in Moderately to Severely Active Ulcerative Colitis Patients From GEMINI 1. Gastroenterology, Vol. 150, Issue 4, S105

24. Stephane Paul, Nicolas Williet, Pierre Claudez, Marion Fauvet, Emilie Del Tedesco, Camille Jarlot, Jean Marc Phelip, Christian Genin, and others. Serum Vedolizumab Assay at Week 6 Predicts Sustained Clinical Remission and Lack of Recourse to Optimisation in IBD. Gastroenterology, Vol. 150, Issue 4, S410

25. Robert Battat, Uri Kopylov, Talat Bessissow, Alain Bitton, Albert Cohen, Myriam Martel, Ernest G. Seidman, Waqqas Afif. Association of Ustekinumab Trough Concentrations With Clinical, Biochemical and Endoscopic Outcomes. Gastroenterology, Vol. 150, Issue 4, S144-S145

26. John B. Kisiel, Hatim T. Allawi, Pasquale Klepp, Tamara Sander, William R. Taylor, Tracy C. Yab, Stephan A. Brackmann, Arne Røseth, and others Novel Stool DNA Markers for Inflammatory Bowel Disease Associated Colorectal Cancer and High Grade Dysplasia: High Specificity Across Three Independent International Populations.. Gastroenterology, Vol. 150, Issue 4, S48 
27. Siew C. Ng, Tsz Wai Lee, Ching Man Tang, Cheuk Lau Wong, Tsz Yiu Lam, Vernon Lam, Harris Ho Hin Lo, Wai K. Leung, and others. Cancer Risk and Predictors in 2,432 Chinese Patients With Inflammatory Bowel Disease: A Population-Based Cohort Study. Gastroenterology, Vol. 150, Issue 4, S6-S7

28. Harminder Singh, Zoann Nugent, Lisa Lix, Laura Targownik, N. Jewel Samadder, Charles N. Bernstein. There Is No Decrease in the Mortality From IBD Associated Colorectal Cancers Over 25 Years: A Population Based Analysis. Gastroenterology, Vol. 150, Issue 4, S226-S227. 
TABLA 1: RESUMEN DE LAS NOVEDADES PRÁCTICAS EN LA DDW 2016 EN EPIDEMIOLOGIA, SEGUIMIENTO, MONITORIZACION Y CONTROL TERAPEUTICO, Y PREVENCIÓN DEL CÁNCER DE COLON EN LA ENFERMEDAD INFLAMATORIA INTESTINAL

\section{¿EXISTE ALGÚN AVANCE RELEVANTE EN LA ETIOLOGÍA O HISTORIA NATURAL DE LA EII?}

No. Apenas hemos avanzado en encontrar la etiología de la Ell. En cuanto a la historia natural de la Ell, un estudio sugiere que en los pacientes con CU estables tras 1 año con tratamiento anti TNF, retirarlo no es recomendable, si bien queda por demostrar de forma firme. Si se retira en algún caso, se recomienda continuar con tiopurinas.

¿DISPONEMOS DE MEJORES FACTORES PREDICTORES DE LA EVOLUCIÓN INDIVIDUAL DE NUESTROS PACIENTES CON EII?

No. Un estudio de valor relativo sugiere que monocitosis o la composición corporal podría ser un factor a tener en cuenta. También parece que los casos de Ell recién diagnosticados hacen un mayor uso de los servicios de urgencias, que quizá podríamos optimizar.

¿QUE HAY DE NUEVO EN EL SEGUIMIENTO / MONITORIZACION DE LA EII Y DE SUS TRATAMIENTOS?

Los niveles de anti TNF son útiles para tomar decisiones en la práctica clínica ante la pérdida de respuesta secundaria, pero no han probado su utilidad como monitorización proactiva o como factor pronóstico. En el caso de la enfermedad perianal podrían ser necesarios niveles más elevados. Disminuir o discontinuar la azatioprina al año de tratamiento combinado (anti TNF y azatioprina), en pacientes estables y en remisión profunda, es una alternativa a explorar.

¿EXISTEN AVANCES PRÁCTICOS IMPORTANTES EN LA PREVENCIÓN DEL CCR EN LA ENFERMEDAD INFLAMATORIA INTESTINAL?

No cambiaremos nuestra práctica clínica de momento. Un nuevo método molecular de detección de ADN metilado en heces parece prometedor en la 
prevención del CCR en la Ell. No obstante, de momento debemos seguir las recomendaciones de las guías actuales como mejor manera de intentar prevenir el CCR en nuestros pacientes con Ell, aunque su aplicación con matices individuales es razonable. Debemos considerar el riesgo de otros tumores en nuestros pacientes con Ell.

\section{Abreviaturas usadas en la tabla 1:}

Ell: enfermedad inflamatoria intestinal.

CU: Colitis UlcerosalFX: Infliximab.

TNF: Tumor Necrosis FactorCCR: cáncer colorrectal

CCR: Cáncer colorrectal

ADN: ácido desoxirribonucleico 